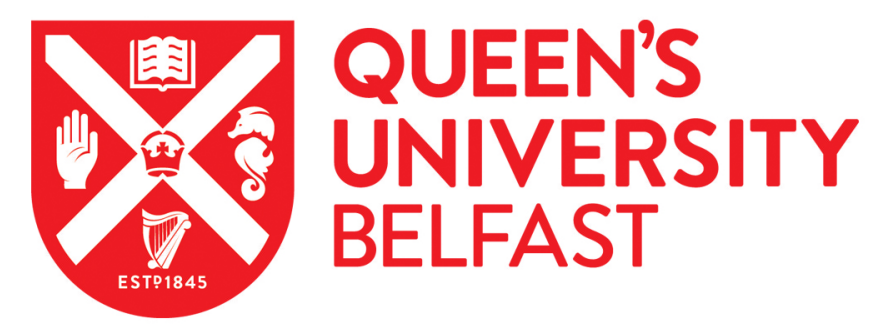

\title{
Modeling Nonlinear Wave Digital Elements using the Lambert Function
}

Bernardini, A., Werner, K. J., Sarti, A., \& Smith, J. O. (2016). Modeling Nonlinear Wave Digital Elements using the Lambert Function. IEEE Transactions on Circuits and Systems I: Regular Papers, 63(8), 1231-1242. https://doi.org/10.1109/TCSI.2016.2573119

\section{Published in:}

IEEE Transactions on Circuits and Systems I: Regular Papers

\section{Document Version:}

Peer reviewed version

\section{Queen's University Belfast - Research Portal:}

Link to publication record in Queen's University Belfast Research Portal

\section{Publisher rights}

( ) 2016 IEEE. Personal use of this material is permitted. Permission from IEEE must be obtained for all other users, including reprinting/ republishing this material for advertising or promotional purposes, creating new collective works for resale or redistribution to servers or lists, or reuse of any copyrighted components of this work in other works

\section{General rights}

Copyright for the publications made accessible via the Queen's University Belfast Research Portal is retained by the author(s) and / or other copyright owners and it is a condition of accessing these publications that users recognise and abide by the legal requirements associated with these rights.

Take down policy

The Research Portal is Queen's institutional repository that provides access to Queen's research output. Every effort has been made to ensure that content in the Research Portal does not infringe any person's rights, or applicable UK laws. If you discover content in the Research Portal that you believe breaches copyright or violates any law, please contact openaccess@qub.ac.uk. 


\title{
Modeling Nonlinear Wave Digital Elements using the Lambert Function
}

\author{
Alberto Bernardini, Student Member, IEEE, Kurt J. Werner, Student Member, IEEE, \\ Augusto Sarti, Senior Member, IEEE, Julius O. Smith III, Member, IEEE
}

\begin{abstract}
A large class of transcendental equations involving exponentials can be made explicit using the Lambert $W$ function. In the last fifteen years, this powerful mathematical tool has been extensively used to find closed-form expressions for currents or voltages in circuits containing diodes. Until now almost all the studies about the $W$ function in circuit analysis concern the Kirchhoff (K) domain, while only few works in the literature describe explicit models for diode circuits in the Wave Digital (WD) domain. However explicit models of NonLinear Elements (NLEs) in the WD domain are particularly desirable, especially in order to avoid the use of iterative algorithms. This paper explores the range of action of the $W$ function in the WD domain; it describes a procedure to search for explicit wave mappings, for both one-port and multi-port NLEs containing diodes. WD models, describing an arbitrary number of different parallel and anti-parallel diodes, a transformerless ring modulator and some BJT amplifier configurations, are derived. In particular, an extended version of the BJT Ebers-Moll model, suitable for implementing feedback between terminals, is introduced.
\end{abstract}

Index Terms-NonLinear Wave Digital Filters, Lambert Function, Diodes, Virtual Analog.

\section{INTRODUCTION}

$\mathbf{W}$ AVE Digital Filter (WDF) theory was introduced by A. Fettweis [1] in the early $70 \mathrm{~s}$ as a method for designing digital filters through the discretization of analog circuits. A WDF is the result of a linear mapping of circuit port variables in the Kirchhoff $(\mathrm{K})$ domain (voltages and currents) onto variables in the Wave Digital (WD) domain (incident and reflected waves). The presence of reactive components in the circuit also requires a bilinear transformation (from the Laplace domain to the $\mathrm{Z}$ domain) in the discretization process. In compliance with circuit theory, components are thought of as connected to each other through ports. A port in the $\mathrm{K}$ domain is characterized by a pair of signals (current $I$ and voltage $V$, respectively). In the WD domain a port is described by a different pair of wave signals (incident wave $a$ and reflected wave $b$, respectively). The mapping from a $\mathrm{K}$ pair onto a WD pair is, in fact, linear and invertible:

$$
\underbrace{a=V+I R_{0}, b=V-I R_{0}}_{\text {K-WD }}, \underbrace{V=\frac{a+b}{2}, I=\frac{a-b}{2 R_{0}}}_{\text {WD-K }},
$$

A. Bernardini and A. Sarti are with the Dipartimento di Elettronica, Informazione e Bioingegneria, Politecnico di Milano, Piazza L. Da Vinci 32, 20133 Milano, Italy (e-mail: [alberto.bernardini,augusto.sarti]@ polimi.it). K.J. Werner and J.O. Smith III are with the Center for Computer Research in Music and Acoustics (CCRMA), Stanford University, Stanford, California 94305-8180, USA (e-mail: [kwerner,jos] @ ccrma.stanford.edu). Copyright (c) 2016 IEEE. Personal use of this material is permitted. However, permission to use this material for any other purposes must be obtained from the IEEE by sending an email to pubs-permissions@ieee.org. where $R_{0}$ is a (non-zero) free parameter called "reference port resistance". Modeling WD networks requires balancing such free parameters in a port-wise fashion, in order to avoid delayfree loops and make the implementation computable.

Despite the initial motivations, the interest in WDF theory has kept steadily strong throughout the decades, as it progressively shifted from filter design to circuit emulation, with numerous new applications to sound synthesis through physical modeling [2] and virtual analog modeling [3]. In the WD domain we can closely simulate the behavior of a reference circuit in a modular and efficient fashion. Moreover, unlike classical circuit simulation tools such as SPICE, WDFs ensure that many of the good properties of the analog reference circuit will be preserved. For example, passivity and losslessness of analog circuits are preserved by their WD implementation [4]. Furthermore, the behavior of a WD implementation will be insensitive to the quantization of coefficients. This means that a WD implementation will be able to offer a good dynamic range with modest accuracy requirements. In addition, the sensitivity properties of WD implementations also guarantee stability under mild conditions, producing structures that tend to exhibit neither limit cycles nor zero-input parasitic oscillations [5]. Finally, this inherent robustness makes working in the WD domain particularly suitable for interactive real-time applications, where model parameters are altered on the fly, as opposed to the off-line simulations performed by SPICE. Moreover, parameter update turns out to be easier using WD Structures (WDSs) w.r.t. other physical modeling approaches developed for real-time applications, such as the nonlinear state-space formulation, as discussed in [6]. As an example of the major complexity of parameter update using nonlinear state-space systems we refer to [7]. Originally conceived for modeling a linear circuit, WDF theory turned out to be suitable also for describing circuits containing NonLinear Elements (NLEs) without and with memory [8], [9]. In particular, relevant examples of nonlinear circuits in the WD domain for virtual analog applications involve diodes [10]-[13], vacuum tubes [14]-[17], BJTs [18], [19] as well as transformers [20].

Most classical WD implementations are tree-like structures (e.g., Binary Connection Tree [21]). The leaves of such trees are one-port linear elements, while the nodes are adaptors. There are also some circuits that exhibit special interconnection topologies resulting in WDSs that are not binary-tree-like. Such cases can be addressed using SPQR graph decomposition [19], [22]-[24], which remaps the WDS into a tree through the introduction of special (R-type) nodes. One inherent weakness of WDSs is that they can only accommodate one NLE at a 
time (see [21]), which must be placed at the root of the tree. Some strategies for overcoming this limitation are described in [3] and consist, for example, of consolidating the NLEs of the reference circuit into a single multi-port NLE. A first example of application of this approach is presented in [17]. The implementation of WD multi-port NLEs, however, very often requires the solution of multidimensional systems of implicit equations, which are very hard to turn into a set of explicit WD equations. A possible remedy, adopted in [17], consists of iteratively finding a solution of the system of equations at every time step. This solution, however, is rather demanding from the computational standpoint, and the convergence of these iterative methods might raise some issues, particularly as the dimensionality of the solution space increases. For these reasons, explicit equations describing the NLE are generally preferable. One method that goes in this direction is proposed in [18] and is based on a piecewise linear approximation of the consolidated NLE. In this work we take a completely different route, seeking exact solutions for a specific class of NLEs.

In circuit theory, there exists a wide class of nonlinearities that exhibits an exponential behavior (e.g., diodes). A good property of many exponential equations is that they can be explicitly solved using the Lambert $W$ function [25]. Banwell and Jayakumar [26] first exploited this property by finding a closed-form expression for the current that passes through a diode in series with a resistance and a voltage generator. Banwell also derived explicit solutions for currents in some circuits containing BJTs [27]. In the WD domain the $W$ function was used in [11] to find a wave mapping of a oneport NLE representing the Shockley diode model. In this manuscript we start from Banwell's approach and explore its applicability to WD circuit models. With this purpose in mind, we define a new family of multi-port exponential NLEs characterized by explicit wave mappings.

Section $\amalg$ defines the $W$ function and focuses on its use in the analysis of diode circuits. Section III describes a procedure for deriving explicit wave mappings of one-port exponential NLEs and presents a new WD model of an arbitrary bank of different parallel and anti-parallel diodes. Section [V] extends the procedure of Section [II] to the multi-port case, by introducing a new type of port that is necessary for modeling multiport elements. We then present a new family of multi-port exponential NLEs and describe some Simplified Models that are useful to widen the class of implementable circuits. Section $\mathrm{V}$ presents some examples of application focused on, but not limited to, audio circuitry, such as the asymmetrical electrical damper (used in envelope generation), the ring modulator (a common audio effect) and three configurations of BJT amplifiers. In particular we introduce an extended version of the Ebers-Moll model of the BJT, which is suitable for implementing the feedback between terminals. Section VI concludes this paper.

\section{LAMbert Function BACKGRound IN DiOde Circuits ANALYSIS}

The so called Lambert function $W(z)$ is a well known set of functions of the complex variable $z$ [25], which is implicitly defined by the exponential equation

$$
z=W(z) e^{W(z)} \quad \forall z \in \mathbb{C} .
$$

In this work we will restrict the domain of the $W$ function, turning $z$ into a real variable $x$, so that also $W(x)$ becomes real. In particular we will exploit an application of the $W$ function proposed in [25], which allows us to find an explicit solution of exponential equations of the form

$$
p^{\theta y+\beta}=c y+d
$$

with $p>0, c, \theta \neq 0, d$ and $\beta$ real parameters and $y$ a real variable. These equations, in fact, can be solved in closed form with respect to the variable $y$ as

$$
y=-\frac{W\left(-\frac{\theta \ln p}{c} p^{\beta-\frac{\theta d}{c}}\right)}{\theta \ln p}-\frac{d}{c} .
$$

For the evaluation of $W(x)$ we suggest the same iterative approach mentioned in [11] and described in [28], as it is suitable for real time circuit simulation purposes. According to such approach we can write

$$
W_{k+1}(x)= \begin{cases}\ln \frac{x}{W_{k}(x)} & \text { if } x \geq e \\ x e^{-W_{k}(x)} & \text { if } x<e\end{cases}
$$

where $W_{k+1}(x)$ is an improved solution w.r.t. $W_{k}(x)$ and $k=0,1,2 \ldots$ is the iteration index. The initial values $W_{0}(x)$ can be precisely computed using mathematical softwares, e.g. MATLAB or Mathematica, and then tabulated in a lookup table with the needed granularity. This iterative approach provides high flexibility in terms of computational complexity, as the number of iterations can be adjusted in order to optimize the trade-off between the desired accuracy and the available computational time. A remarkable advantage in using $W(x)$ in exponential circuit models is that we can use the same lookup table for many different nonlinearities, minimizing the required storage resources.

\section{A. Applications in the $K$ Domain}

The $W$ function made its first appearance in circuit theory with the work of Banwell and Jayakumar [26], which derived a closed-form expression of the current flowing through a diode connected to a voltage source $V_{E}$ and a series resistance $R_{E}$, as in Fig. 4(a) In the Shockley model the relationship between diode current $I$ and voltage $V$ is

$$
I=I_{s}\left(e^{\frac{V}{\eta V_{t}}}-1\right)
$$

where $e$ is Napier's number, $V_{t}$ is the thermal voltage, $\eta$ is the ideality factor and $I_{s}$ is the saturation current of the diode. The problem approached in [26] was finding a closed-form expression for the current $I$, which satisfies both the Shockley model (6) and the $\mathrm{K}$ law $V=V_{E}-R_{E} I$. The solution

$$
I=\frac{\eta V_{t}}{R_{E}} W\left(\frac{I_{s} R_{E}}{\eta V_{t}} e^{\frac{V_{E}+R_{E} I_{s}}{\eta V_{t}}}\right)-I_{s},
$$

proposed in [26], is usually referred to as Generalized Diode Equation (GDE). This result was later extended by Banwell by considering $V_{E}$ and $R_{E}$ as a generic resistive Thévenin 


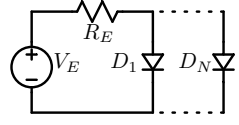

Fig. 1. Multi-exponential Junction Model.

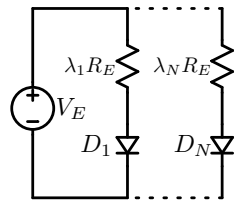

Fig. 2. Alternative Multi-exponential Junction Model. equivalent [27]. He considered some BJT circuits, whose linear parts can be reduced to Thévenin equivalents, and found analytical solutions.

The study of circuits with diodes is quite common in the photovoltaic field, as the typical models for ideal solar cells involve a parallel bank of diodes, representing the silicon $\mathrm{p}-\mathrm{n}$ junction, in parallel with a current source. In order to describe real solar cells, a series resistance and a parallel shunt resistance are often added to the model. This is, therefore, one case where the GDE can be put into use. The GDE was, in fact, applied to the solar cell model in [29] and an explicit solution for the current passing through a single exponential junction with a parasitic series and two parasitic parallel shunt resistances was found. Following these results, techniques for estimating solar cell parameters exploiting the $W$ function [30]-[32] were developed. New analytical methods for extracting diode parameters [33], [34] were proposed shortly after.

Real semiconductor $\mathrm{p}-\mathrm{n}$ junctions, are known to exhibit multiple simultaneous conduction mechanisms, usually described with multi-exponential models, characterized by a parallel bank of diodes with different ideality factors and saturation currents as shown in [35], [36]. In these works the junction model with $N$ conduction mechanisms presented in Fig. 1 is approximated by the alternative model in Fig. 2. The global Thévenin resistance $R_{E}$ is replaced by $N$ individual series resistances $\lambda_{n} R_{E}$ being $1 \leq n \leq N$. $\lambda_{n}$ are positive real parameters that must be tuned carefully in order to obtain the best approximation. The alternative model is equivalent to the original one only if all the diodes are identical and we set $\lambda_{n}=N$ for each branch. In the other cases, however, we can still obtain good approximations. Using the alternative model, an exact closed-form solution for the currents passing through the diodes can be found [35], [36]. The resistances $\lambda_{n} R_{E}$ allow us to compute the currents passing through each individual branch using the GDE (7). The individual currents can then be summed in order to find the explicit global solution

$$
I=\sum_{n=1}^{N} \frac{\eta_{n} V_{t}}{\lambda_{n} R_{E}} W\left(\frac{I_{s_{n}} \lambda_{n} R_{E}}{\eta_{n} V_{t}} e^{\frac{V_{E}+\lambda_{n} R_{E} I_{s_{n}}}{\eta_{n} V_{t}}}\right)-I_{s_{n}} .
$$

\section{B. Applications in the WD Domain}

Not much effort has been devoted to the development of applications of the $W$ function in the WD domain. In [11] an explicit one-port WD version of the Shockley model (6) is obtained substituting (1) in (6) and using the $W$ function:

$$
b=a+2 R_{0} I_{s}-2 n V_{t} W\left(\frac{R_{0} I_{s}}{n V_{t}} e^{\frac{R_{0} I_{s}+a}{n V_{t}}}\right) .
$$

In addition, in [11] a one-port diode clipper model containing two identical anti-parallel diodes is presented, where only one of the two diodes is conducting at any time. This way the port current can be approximated with the forward current passing through the conducting diode, while ignoring the reverse leakage current of the other diode. The resulting wave mapping is

$$
b=\operatorname{sgn}(a)\left(|a|+2 R_{0} I_{s}-2 n V_{t} W\left(\frac{R_{0} I_{s}}{n V_{t}} e^{\frac{R_{0} I_{s}+|a|}{n V_{t}}}\right)\right),
$$

where $\operatorname{sgn}(a)$ is a function returning the sign of $a$ and suggests which diode is conducting. In [37] an improvement of this model of the diode clipper is proposed. Both in [11] and in [37] the diodes characterizing the NLE are assumed to be equal (i.e., same ideality factor and saturation current). In this paper we will discuss the more general case where diodes are not necessarily identical.

\section{EXPLICIT WD MODELS OF ONE-PORT NLES}

In order to search for an explicit wave mapping that describes a one-port NLE containing exponentials, we can start from its description in the $\mathrm{K}$ domain and then repurpose it in the WD domain. One common way to do so is writing the $\mathrm{K}$ port variables directly as (1), but often this substitution leads to complicated systems to solve. One general procedure for determining explicit wave mappings in a straightforward fashion consists of three main steps. We first write the port current, or the port voltage, as

$$
I=\frac{a-V}{R_{0}}, \quad V=a-R_{0} I .
$$

We then rearrange the system, searching an equation that fits the general form (3) presented in [25]. The unknown variable $y$ can either be $V$ or $I$, depending on how the substitution was performed. If we succeed in turning the system in the form (3), we obtain an explicit solution for $y$ using (4). We can finally compute the reflected wave using

$$
b=2 V-a \quad \text { or } \quad b=a-2 R_{0} I .
$$

When dealing with one-port NLEs we can often resort to exploiting what we refer to as the "K Domain Analogy" (KDA), which consists of replacing the circuit that the NLE connects to with its Thévenin equivalent, and deriving from this simplified representation the corresponding wave mapping. More specifically, if $V_{E}$ is the voltage generator of the Thévenin equivalent and $R_{E}$ its series resistance, as shown in Fig. 4, setting $V_{E}=a$ and $R_{E}=R_{0}$, the $\mathrm{K}$ equations describing the one-port element in series to the Thévenin equivalent become identical to (11). Therefore, if we already have $\mathrm{K}$ equations expressing $I$ or $V$ of a one-port NLE in series to a Thévenin equivalent, we could skip the first two steps of the procedure and derive the correspondent wave mapping, by simply setting $V_{E}=a, R_{E}=R_{0}$ and then using 12.

\section{A. Derivation of the Diode Model using the KDA}

Let us now apply the KDA to the same circuit that was analyzed in [26]. Starting from the GDE (7), we derive an 
explicit equation, expressing the port voltage $V$ as a function of the incident wave $a=V_{E}$ and the reference port resistance $R_{0}=R_{E}$ :

$$
V=a-\eta V_{t} W\left(\frac{I_{s} R_{0}}{\eta V_{t}} e^{\frac{a+R_{0} I_{s}}{\eta V_{t}}}\right)+R_{0} I_{s} .
$$

Finally, substituting (13) in (12), we obtain exactly the same result presented in [11] (eq. (9)) while skipping all the algebra needed in the second step of the procedure.

\section{B. Parallel and Anti-Parallel Bank of Diodes Model}

We apply the KDA to the multi-exponential model represented in Fig. 1. where the NLE is a parallel bank of $N$ diodes. As discussed in [35] and [36] we cannot compute the global current $I$ of the model in Fig. 1 1 with explicit equations, but we can do it using the alternative model in Fig. 2. So starting from equation (8), we easily derive the following wave mapping:

$$
b=a-2 R_{0} \sum_{n=1}^{N}\left[\frac{\eta_{n} V_{t}}{\lambda_{n} R_{0}} W\left(\frac{\lambda_{n} R_{0} I_{s_{n}}}{\eta_{n} V_{t}} e^{\frac{a+\lambda_{n} R_{0} I_{s_{n}}}{\eta_{n} V_{t}}}\right)-I_{s_{n}}\right]
$$

where $\eta_{n}$ and $I_{s_{n}}$ are the ideality factor and the saturation current of the $n$th diode, $R_{0}$ is the reference port resistance and $\lambda_{n}$ is its multiplicative factor relative to the $n$th branch. When $N=1$ and $\lambda_{1}=1$, the scattering equation (14) coincides with (9). Notice that this result could not have been obtained by blindly applying the three-step-procedure described at the beginning of this Section, as any of the terms that play the role of $y$ in eq. (3) are neither port currents nor port voltages. In this case, in fact, the " $y$ variables" are the currents passing through each diode branch, which all contribute to the global port current of the NLE. This, however, does not affect the generality of the three-step-procedure as its validity relies on the fact that $R_{0}$ is split into $N$ series resistances $\lambda_{n} R_{0}$ to begin with. Both a parallel bank of diodes, such as the one in Fig. 1, and a parallel bank of diodes with a series resistance in each branch, such as the one in Fig. 2, can be described in the WD domain by the wave mapping (14).

Combining eq. (14) with eq. (10), we derive an even more general wave mapping describing an arbitrary number of different parallel and anti-parallel diodes:

$$
\begin{aligned}
& b=a-2 R_{0} \Theta_{+}(a) \sum_{n=1}^{N} \frac{\eta_{n} V_{t}}{\lambda_{n} R_{0}} W\left(\frac{\lambda_{n} R_{0} I_{s_{n}}}{\eta_{n} V_{t}} e^{\frac{|a|+\lambda_{n} R_{0} I_{s_{n}}}{\eta_{n} V_{t}}}\right)-I_{s_{n}} \\
& -2 R_{0} \Theta_{-}(a) \sum_{m=N+1}^{N+M} \frac{\eta_{m} V_{t}}{\lambda_{m} R_{0}} W\left(\frac{\lambda_{m} R_{0} I_{s_{m}}}{\eta_{m} V_{t}} e^{\frac{|a|+\lambda_{m} R_{0} I_{s_{m}}}{\eta_{m} V_{t}}}\right)-I_{s_{m}}
\end{aligned}
$$

where $\Theta_{+}(a)$ and $\Theta_{-}(a)$ are two functions defined as

$$
\Theta_{+}(a)=\left\{\begin{array}{l}
1 \text { if } a \geq 0 \\
0 \text { if } a<0
\end{array} \quad \Theta_{-}(a)=\left\{\begin{array}{ll}
0 & \text { if } a \geq 0 \\
-1 & \text { if } a<0
\end{array} .\right.\right.
$$

The NLE described by eq. (15) is depicted in Fig. 3 If all diodes are identical, $\lambda_{n}=N$ and $\lambda_{m}=M$, 15 reduces to (10). In general, the wave mapping (15) should be used carefully in extreme cases in which $N \gg M$ or $M \ll N$, as the reverse bias saturation currents might be not negligible. By the way, (15) could be used for deriving a WD model of a solar cell with a multi-exponential junction [35], [36]. In Section $\mathrm{V}$ other possible applications of (15) are shown.

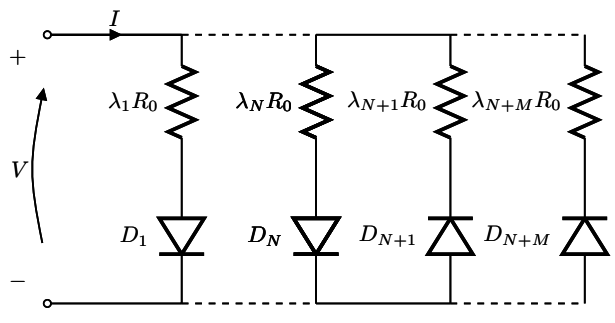

Fig. 3. One-Port NLE characterized by a Parallel Bank of $N+M$ Diodes with Opposite Polarities.

\section{Explicit WD Models OF MUlti-Port NLES}

In this Section we focus on the modeling of multi-port NLEs. In particular, we present an extension to the multiport case of the procedure proposed in Section III for deriving a new family of NLEs in the WD domain (using explicit wave mappings). We finally offer a definition of Simplified Models, which are approximations of circuits containing nonconducting diodes. These models can be useful to widen the class of circuits that can be implemented in the WD domain.

\section{A. Sign Conventions for Port Variables in n-terminal NLEs}

Each port of a generic NLE has two prongs, which share the same value of current (the current through one prong equals the current through the other one), called port current, which has a well-defined reference orientation. In this work we define the coupled port voltage variable according to the passive sign convention as shown in Fig. 3. In traditional one-port NLEs the port current enters the NLE and passes through it. When it comes to defining the ports of a multi-terminal NLE, the situation can easily become more complicated. Instead of defining waves with reference to the NLE, it will often be more practical to define them with reference to the load that is connected to the NLE, rather than with reference to the NLE itself. This results in a change of the reference orientation of the port current as it enters the load instead of the NLE. The change of the reference orientation of the port current implies a change of the roles of the incident and the reflected waves ( $a$ and $b$ ). For this reason we will need to flip the sign of the waves when defining the port current. This fact was already pointed out in [17]. In order to clarify this, let us first consider the simplest case of a circuit with a one-port NLE in Fig. 4(a) and the relative WD implementation in Fig. 4(b) This is a configuration where port variables can be defined with reference the NLE (port current entering the NLE). When port variables are defined this way, we will refer to the port as a Port of the First Type (PFT). The 3-terminal NLE in Fig. 5(a) on the other hand, cannot be easily described using PFTs. In this case it is more convenient to define port variables with reference to the loads that are connected to the NLE, i.e., to pick the reference port currents as entering such loads, as done in [17]. This means interpreting the port configuration as shown in Fig. 5(b) As we can see, two of such ports connect to each other through a prong (ground), which is not a terminal of the NLE. When a port current is defined as directed outward with respect to the NLE, as in the case of Fig. 5(a), we will 
talk about a Port of the Second Type (PST). While the two prongs of a PFT are always, by definition, terminals of the NLE that we are modeling, in the case of a PST one of the two prongs might not be a terminal of the NLE. In this case it is often useful to choose ground as common external prong of many ports. We can deduce, therefore, that the number of ports $N$ of a generic $n$-terminal NLE with $n>2$ may vary according to the topology of the reference circuit in the range $1 \leq N \leq N_{\max }$, where

$$
N_{\max }(n)=\frac{n(n+1)}{2}=\left(\begin{array}{c}
n+1 \\
2
\end{array}\right) .
$$

In the light of this in Section $\mathrm{V}$ we will present a novel model for a generic 3-terminal BJT with $N_{\max }(3)=6$ ports 31 .

In order to accommodate PFTs and PSTs we will adopt the following notation for port voltages and port currents:

$$
V=\frac{a+b}{2} \quad I=\delta_{p} \frac{a-b}{2 R_{0}}
$$

where $\delta_{p}=1$ when in the case of a PFT and $\delta_{p}=-1$ in the case of a PST. In general, a NLE is modeled using either all PFTs or PSTs.

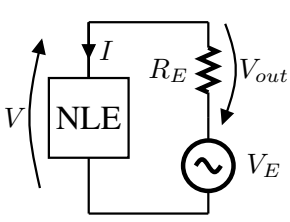

(a) Schematic

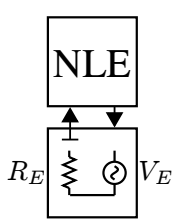

(b) WDS

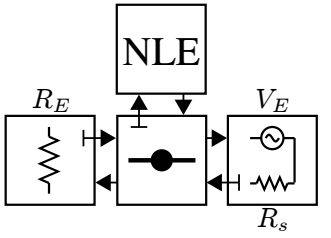

(c) WDS
Fig. 4. One-port NLE modeled using one PFT. The WDS in 4(b) refers to Subsection IV-A The WDS in 4(c) refers to Subsection V-A

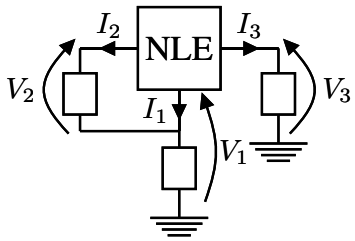

(a)

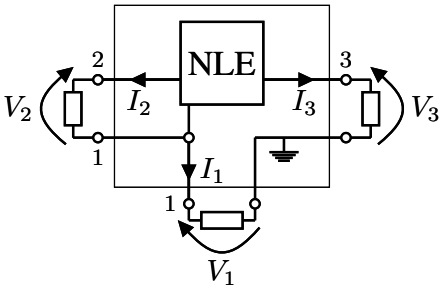

(b)
Fig. 5. 5(a) shows a 3-terminal NLE that cannot be easily modeled using 3 PFTs. In 5(b) we have the same NLE shown in 5(a) modeled using three PSTs; two of such ports have a common external prong (ground), which is not one of the 3 terminals of the NLE.

\section{B. WD Multi-port NLEs Derivation}

In this Subsection we will describe how to derive WD exponential and explicit multi-port models. We will begin with the simplest case of a NLE made of only one subcircuit and then we will address the general case of a NLE made of $K$ subcircuits that can be independently analyzed. For instance, the circuit in Fig. 7 has a NLE containing $K=2$ independently analyzable subcircuits, which are the two separated diodes.

Let us first consider the simplest case of a single subcircuit and assume that the whole NLE be connected to the rest of the circuit through $N$ ports, which in this Subsection are indicated with integers $1,2, \ldots, N$. Let us derive a system of equations describing the subcircuit in the $\mathrm{K}$ domain. We then express the Kirchhoff variables of the generic port $x$ with $1 \leq x \leq N$, using one of the two formulas:

$$
I_{x}=\delta_{p_{x}} \frac{a_{x}-V_{x}}{R_{0 x}}, \quad V_{x}=a_{x}-\delta_{p_{x}} R_{0 x} I_{x} .
$$

We refer to the unknown variable as $y$, which is either a port voltage or a port current. In order to find explicit wave mappings we need to express each port variable as a linear function of $y$. If the unknown variable is a port voltage, e.g. $y=V_{1}$, then each port voltage $V_{x}$ must satisfy the equation

$$
V_{x}=\tau_{x} V_{1}+\rho_{x},
$$

where $\tau_{x}$ and $\rho_{x}$ are scalar functions of the incident waves and the reference port resistances, i.e. they can be written as $\tau_{x}\left(a_{1}, \ldots, a_{N}, R_{01}, \ldots, R_{0 N}\right)$ and $\rho_{x}\left(a_{1}, \ldots, a_{N}, R_{01}, \ldots, R_{0 N}\right)$. Similarly, if the unknown variable is a port current, e.g. $y=I_{1}$, then each port current $I_{x}$ must satisfy the equation

$$
I_{x}=\zeta_{x} I_{1}+\nu_{x},
$$

where, again, $\zeta_{x}$ and $\nu_{x}$ are scalar functions, which can be written as $\zeta_{x}\left(a_{1}, \ldots, a_{N}, R_{01}, \ldots, R_{0 N}\right)$ and $\nu_{x}\left(a_{1}, \ldots, a_{N}, R_{01}, \ldots, R_{0 N}\right)$.

By defining $\boldsymbol{V}=\left[V_{1}, \ldots, V_{N}\right]^{T}, \boldsymbol{\tau}=\left[1, \tau_{1}, \ldots, \tau_{N}\right]^{T}$, $\boldsymbol{\rho}=\left[0, \rho_{1}, \ldots, \rho_{N}\right]^{T}, \boldsymbol{y}=\left[V_{1}, 1\right]^{T}$, we obtain a matrix formulation of the mutual relations between port voltages:

$$
\boldsymbol{V}=\left[\begin{array}{ll}
\boldsymbol{\tau} & \rho
\end{array}\right] \boldsymbol{y} .
$$

Similarly, by defining $\boldsymbol{I}=\left[I_{1}, \ldots, I_{N}\right]^{T}, \quad \boldsymbol{\zeta}=$ $\left[1, \zeta_{1}, \ldots, \zeta_{N}\right]^{T}, \boldsymbol{\nu}=\left[0, \nu_{1}, \ldots, \nu_{N}\right]^{T}, \boldsymbol{y}=\left[I_{1}, 1\right]^{T}$, we obtain:

$$
I=\left[\begin{array}{ll}
\zeta & \nu
\end{array}\right] \boldsymbol{y} .
$$

Then, starting from the derived mutual relations between port variables, we search an equation fitting the form (3), analogously to what done in the one-port case III This will allow us to obtain an explicit solution for $y$ in the form (47). Let us define the vector of the incident waves as $\boldsymbol{a}=\left[a_{1}, \ldots, a_{N}\right]^{T}$; the vector of the reflected waves as $\boldsymbol{b}=\left[b_{1}, \ldots, b_{N}\right]^{T}$; and the diagonal matrix of reference port resistances $\boldsymbol{R}_{\mathbf{0}}=\operatorname{diag}\left(R_{01}, \ldots, R_{0 N}\right)$. Writing $\boldsymbol{b}$ as a function of $\boldsymbol{V}$ or $\boldsymbol{I}$ we obtain, respectively,

$$
\boldsymbol{b}=2 \boldsymbol{V}-\boldsymbol{a} \quad \text { and } \quad \boldsymbol{b}=\boldsymbol{a}-\delta_{p} 2 \boldsymbol{R}_{\mathbf{0}} \boldsymbol{I} .
$$

Let us now consider the more general case in which the NLE is constituted of $K$ independently analyzable subcircuits as, for instance, the NLE in Fig. 7 where $K=2$. In this case the derivation described before would be applied separately for each subcircuit. Let us assume that each subcircuit has $N_{k}$ ports, so that the total number of ports of the NLE is $\sum_{k=1}^{K} N_{k}$. In general, we will end up with $N$ explicit scattering vector functions in one of the two forms (23). 


\section{Simplified Models of NLEs with diodes}

Simplified Models (SMs) approximate the behavior of NLEs eliminating diodes which are "not conducting" (i.e., which ones are currently not operating in the first quadrant of their $I V$ characteristics). Such models are based on what we know about the mutual relations between terminal potentials. Thanks to this simplification we can transform many NLEs that could not be modeled with explicit wave mappings into something that now belongs to the family of NLEs described in Subsection IV-B In order for this approach to be effective, we might need to approximate the target NLE with different SMs, which take turn depending on how the mutual relations between the potentials of the terminals change. An example

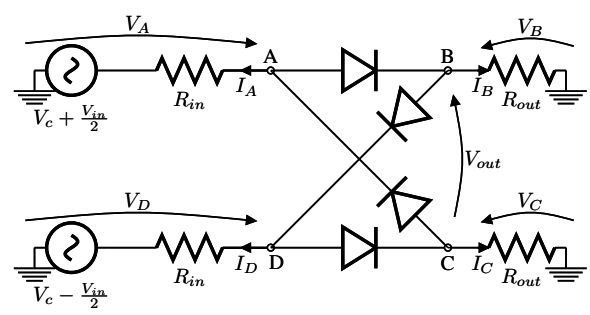

Fig. 6. Parker's Model of a Transformer-less Ring Modulator. The output voltage is $V_{\text {out }}=V_{B}-V_{C}$.

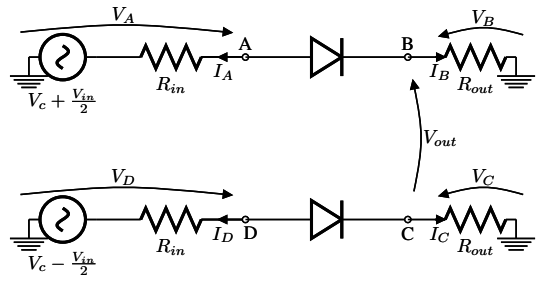

(a) Schematic

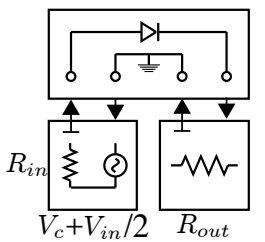

(b) WDS
Fig. 7. Ring Modulator SM for $V_{c}>0$. The WDS in Fig. 7(b) refers only to the subcircuit with the generator $V_{c}+V_{i n} / 2$. The complementary WDS referring to the subcircuit with $V_{c}-V_{i n} / 2$ is similar.

of use of SMs is the transformerless ring modulator model of Fig. 6 [38]. In a traditional ring modulator, if we denote the modulator waveform by $V_{i n}$ and the carrier waveform by $V_{c}$, the voltage at the two input nodes is given by $V_{c}+V_{i n} / 2$ and $V_{c}-V_{i n} / 2$. The circuit of Fig. 6 can be approximated using two different SMs. The first model (Fig. 7) describes the ring modulator when $V_{c}>0$, in which case we remove the nonconducting diodes $D_{C A}$ and $D_{B D}$. Similarly, when $V_{c}<0$, we remove $D_{A B}$ and $D_{D C}$. This, indeed, is an approximation because the actual voltages applied to the diodes are also affected by the contribution of both $V_{i n} / 2$ and the resistive voltage loss on $R_{i n}$. However, in this particular example, we can assume $V_{i n} \ll V_{c}$, therefore this approximation is expected not to significantly affect the circuit behavior.

While identifying SMs is readily done through visual circuit inspection, the selection of which SM is active during the WD simulation might not be as straightforward. The problem, in fact, is to select which SM is active at any time using just the values of incident waves $\boldsymbol{a}$. The state of activation of a diode depends on the sign of the voltage at its terminals, which must be derived from the port voltages $\boldsymbol{V}$, which, in turn, must be derived from $\boldsymbol{a}$. As $\boldsymbol{V}$ generally depends on $\boldsymbol{a}$ and $\boldsymbol{b}$, this might prove tricky. However, in some cases, the information we need on $\boldsymbol{V}$ can be derived from properties of $\boldsymbol{a}$ only, e.g. $\operatorname{sgn}(\boldsymbol{a})$. Examples are the ring modulator WD model in Section $\mathrm{V}-\mathrm{C}$ and the one-ports characterized by the wave mappings 10 and 15 A similar discussion about one-port NLEs is also given in [37] where a diode-clipper is analyzed. Here we extend that discussion to the multi-port case.

In general, the $\mathrm{K}-\mathrm{WD}$ map for a generic $N$-port element can be written in matrix form as

$$
\left[\begin{array}{l}
\boldsymbol{a} \\
\boldsymbol{b}
\end{array}\right]=\boldsymbol{T}\left[\begin{array}{l}
\boldsymbol{V} \\
\boldsymbol{I}
\end{array}\right] \quad \text { with } \boldsymbol{T}=\left[\begin{array}{cc}
\boldsymbol{E}_{N} & \boldsymbol{R}_{\mathbf{0}} \\
\boldsymbol{E}_{N} & -\boldsymbol{R}_{\mathbf{0}}
\end{array}\right],
$$

where $\boldsymbol{E}_{N}$ is the $(N \times N)$ identity matrix. The K-WD transformation 24, characterized by matrix $\boldsymbol{T}$ is, in fact, independent of the internal complexity of the multi-port element, as it is performed in a port-wise fashion. Therefore, considering the $I V$ characteristics at each port, we can use (24) to check if the mutual relations between elements of the vector $\boldsymbol{V}$ are mapped onto corresponding mutual relations between elements of the vector $\boldsymbol{a}$. Similarly to what we did in [37] for the oneport case, we provide a geometric interpretation of the K-WD transformation, which can be useful to relate $\boldsymbol{a}$ to the needed information on $\boldsymbol{V}$. If we apply the QR decomposition on $\boldsymbol{T}$ we obtain $\boldsymbol{T}=\boldsymbol{Q} \boldsymbol{R}$, where the two $(2 N \times 2 N)$ matrices $\boldsymbol{Q}$ and $\boldsymbol{R}$ are generally of the form

$$
\boldsymbol{Q}=\left[\begin{array}{cc}
\frac{1}{\sqrt{2}} \boldsymbol{E}_{N} & \frac{-1}{\sqrt{2}} \boldsymbol{E}_{N} \\
\frac{1}{\sqrt{2}} \boldsymbol{E}_{N} & \frac{1}{\sqrt{2}} \boldsymbol{E}_{N}
\end{array}\right] \quad \boldsymbol{R}=\left[\begin{array}{cc}
\sqrt{2} \boldsymbol{E}_{N} & \boldsymbol{O}_{N} \\
\boldsymbol{O}_{N} & -\sqrt{2} \boldsymbol{R}_{\mathbf{0}}
\end{array}\right]
$$

where $\boldsymbol{O}_{N}$ is an $(N \times N)$ matrix of zeros, therefore $\boldsymbol{R}$ is diagonal (as $\boldsymbol{R}_{0}$ is diagonal as well). Through $(25)$ we can interpret the K-WD transformation as a non-uniform scaling of the $N$ characteristics (through the matrix $\boldsymbol{R}$ ) followed by a rotation of $-\pi / 4$ (through the matrix $Q$ ), which is independent of the port resistance values. If a priori techniques are not sufficient for inferring which diodes are conducting, on line methods should be developed, based on the past simulation parameters. However the formalization of general methods for deriving such information is beyond the scope of this work and deserves future research.

\section{EXAMPLES OF APPLICATIONS}

In this Section we propose some implementations of typical circuits containing diodes or BJTs. The first two examples are possible applications of the new one-port NLEs presented in III-B. The other examples show different multi-port NLEs belonging to the new family of NLEs described in IV-B. where Simplified Models (SMs) are exploited. In particular, we discuss the case of a transformerless ring modulator and some BJT amplifier configurations, which take advantage of a new 6-port general model of the BJT. This is a model that is suitable for dealing with feedback between the BJT terminals. In each simulation we evaluate $W(x)$ using the MATLAB function lambertw and we use a sample rate of $96 \mathrm{kHz}$. 


\section{A. Half-Wave Rectifier with $N$ Different Parallel Diodes}

We employ the NLE depicted in Fig. 2 and characterized by the wave mapping (14) for designing a parametric HalfWave Rectifier (HWR) with a bank of different parallel diodes. The simple reference circuit, consisting of a real sinusoidal voltage generator $V_{E}(t)=g_{E}\left(\sin \left(2 \pi f_{0 E} t\right)\right)$ in series with a resistance $R_{E}=8 \Omega$ and the one-port NLE, is shown in Fig. 4(a) The NLE is modeled using one PFT. In this Subsection we assume the real voltage generator $V_{E}$ has an internal series resistance $R_{s}$. The WD implementation is drawn in Fig. 4(c) In order to accurately model the NLE, we need to properly select the $\lambda_{n}$ parameters in (14). The simplest situation is when the $N$ diodes are identical. In this case, as already mentioned in Subsection [III-B, we should set $\lambda_{n}=N$. In more complex scenarios the $N$ parallel diodes have different parameter values. As an example, we set $N=2, R_{s}=3 \Omega, R_{E}=1 \Omega, g_{E}=10 \mathrm{~V}, f_{0 E}=80$ $\mathrm{Hz}, I_{s_{1}}=I_{s_{2}}=10^{-12} \mathrm{~A}$ and different ideality factors; $\eta_{1}=0.5$ an $\eta_{2}=1$. Experimentally we found that, setting $\lambda_{1}=\lambda_{2}=1.892$, the WD model grasps the circuit behavior. Fig. 8 shows the comparison between the WD and SPICE output voltage signals.

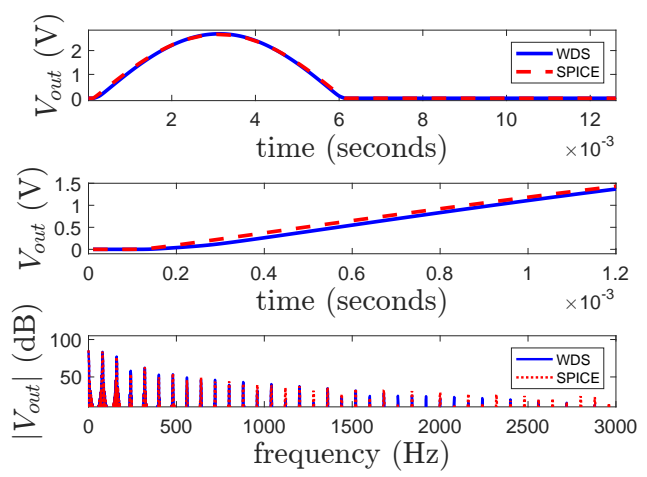

Fig. 8. HWR output voltage (WDS vs SPICE). The upper subplot shows the profile of $V_{\text {out }}$ in the time domain. The subplot in the middle shows a zooming of the same curve near the zero-axis, where the nonlinear behavior is more pronounced. The last subplot represents $V_{o u t}$ in the frequency domain.

\section{B. Electrical Damper}

We can use 15] to implement an electrical damper with different attack and release time constants. The reference

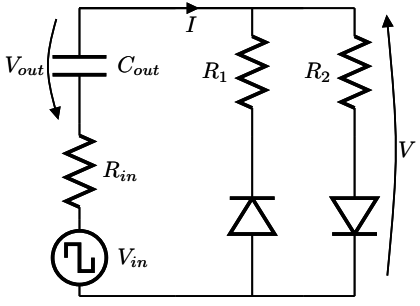

(a) Schematic

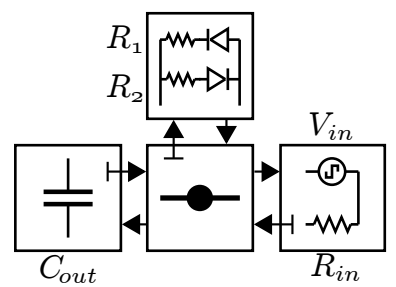

(b) WDS
Fig. 9. Asymmetric Electrical Damper.

circuit and the relative WDS are represented in Fig. 9 A WD implementation of the electrical damper, realized with a different approach, was already presented in [10]. In our implementation diodes and their series resistances are embedded in the NLE. The NLE is modeled using one PFT. The output voltage is detected across the capacitor $C_{i n}$ in series to the NLE. The square wave of the input voltage generator is defined as $V_{\text {in }}(t)=V_{\text {bias }}+g_{\text {in }}\left(\operatorname{sgn}\left(\sin \left(2 \pi f_{0 i n} t\right)\right)\right)$. The coefficients $\lambda_{N}$ and $\lambda_{M}$, which multiply the reference port resistance of the NLE, are tuned in order to match as accurately as possible the resistances $R_{1}$ and $R_{2}$, which in our reference circuit are $4 \Omega$ and $1 \Omega$, respectively. The circuit parameters and the corresponding actual values of the WD simulation are: $g_{i n}=5$ $\mathrm{V}, f_{0 i n}=80 \mathrm{~Hz}, C_{i n}=1.810^{-4} \mathrm{~F}, \eta_{N}=1, \eta_{M}=1$, $V_{\text {bias }}=-6 \mathrm{~V}, \lambda_{n 1}=3.3, \lambda_{m 1}=1.44, V_{t}=2510^{-3} \mathrm{~V}$, $I_{s_{N}}=10^{-12} \mathrm{~A}$ and $I_{s_{M}}=10^{-12} \mathrm{~A}$. Fig. 10 shows the output voltage profile; the good behavior of the WDS is verified comparing it to a SPICE simulation.
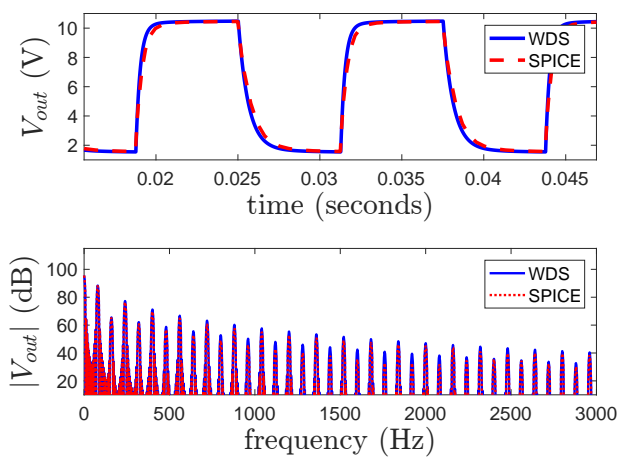

Fig. 10. Asymmetric Electrical Damper output voltage in the time domain and in the frequency domain (WDS vs. SPICE).

\section{Ring Modulator}

In this Subsection we present an implementation of the ring modulator characterized by higher accuracy w.r.t. the existing solutions in the literature on WDFs [18]. We model the NLE using 4 PSTs, named $A, B, C$ and $D$. The port voltages are the four terminal potentials: $V_{A}, V_{B}, V_{C}$ and $V_{D}$. We use two SMs; one for $V_{c}>0$ and one for $V_{c}<0$. Hereafter we analyze in detail the subcircuit of the first SM (Fig. 7) containing diode $D_{A B}$. The subcircuit is described by the system of equations:

$$
\left\{\begin{array}{l}
I_{B}=I_{s}\left(e^{\frac{V_{A B}}{\eta V_{t}}}-1\right) \\
I_{A}=-I_{B} .
\end{array}\right.
$$

Let us choose $I_{B}=y$ as independent port variable; therefore, according to (20), we can express the port current $I_{A}$ as a linear function of $I_{B}$ by writing $I_{A}=-I_{B}$. So in this example the parameters of equation (20) are simply $\zeta_{B}=-1$ and $\nu_{B}=0$. Writing port voltages as functions of incident waves, port currents and port resistances, according to (18), we obtain:

$$
I_{B}=I_{s}\left(e^{\frac{a_{A}-a_{B}-I_{B}\left(R_{0 A}+R_{0 B}\right)}{\eta V_{t}}}-1\right) .
$$

Eq. 27] is attributable to the form [3], setting

$$
\begin{aligned}
& y=I_{B} \quad \theta=-\left(R_{0 A}+R_{0 B}\right) /\left(\eta V_{t}\right) \quad p=e \\
& \beta=\left(a_{A}-a_{B}\right) /\left(\eta V_{t}\right) \quad c=1 / I_{s} \quad d=1 .
\end{aligned}
$$


Then through (4) we derive an explicit expression for $I_{B}$. The wave mappings for ports $A$ and $B$ are found using (23)

$$
b_{B}=a_{B}+2 R_{0 B} I_{B} \quad, \quad b_{A}=a_{A}+2 R_{0 A} I_{A} .
$$

The wave mappings for ports $C$ and $D$ are derived likewise. The equations characterizing the complementary SM are similar to those already shown. The choice of the most suitable SM at each iteration step is performed according to the considerations explained in Section IV-C When $V_{c}$ approaches to 0 the signs of $a_{A}$ and $a_{D}$ may not agree. In this case the sign of one incident wave is chosen, e.g. $a_{A}$, and a small error is introduced. The smaller the magnitude of $V_{i n}$ w.r.t. the amplitude of $V_{c}$, the smaller the error. In our simulation we chose the circuit parameters in such a way to obtain comparable results to [38]: $g_{\text {in }}=1 \mathrm{~V}, f_{0 i n}=500$ $\mathrm{Hz}, R_{\text {in }}=80 \Omega, g_{c}=1 \mathrm{~V}, f_{0 c}=1500 \mathrm{~Hz}, R_{\text {out }}=10^{6} \Omega$, $\eta=2.19, I_{s}=10^{-12} \mathrm{~A}$ and $V_{t}=2610^{-3} \mathrm{~V}$. Notice that $V_{i n}(t)=g_{i n}\left(\sin \left(2 \pi f_{0 i n} t\right)\right)$ and $V_{c}(t)=g_{c}\left(\sin \left(2 \pi f_{0 c} t\right)\right)$, therefore with this choice of parameters the condition of having $V_{i n} \ll V_{c}$ is not satisfied. Nonetheless, even in these conditions, the WD model shows a good agreement with a SPICE simulation, as shown in Fig. 11. The mismatch is concentrated near zero crossings where the switching between SMs takes place. In addition SMs ignore the reverse-bias currents, which are not negligible near the zero-axis.
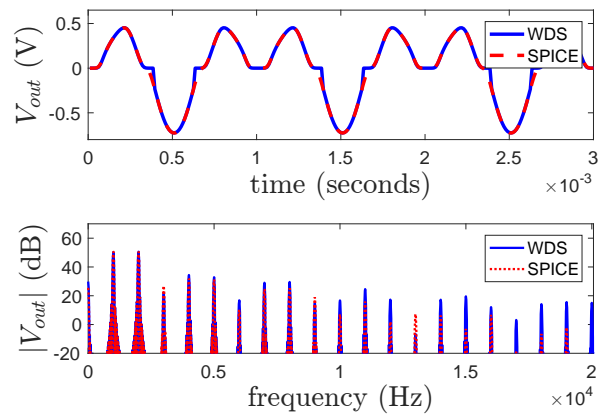

Fig. 11. Ring Modulator output voltage in the time domain and in the frequency domain (WDS vs. SPICE).

\section{Bipolar Junction Transistor Amplifiers}

For modeling an $\mathrm{n}-\mathrm{p}-\mathrm{n}$ BJT we refer to the large signal Ebers-Moll model (EMM) [39] of Fig. 12. We choose the reference direction of currents $I_{C}, I_{E}$ and $I_{B}$, referring to Collector, Emitter and Base terminals respectively, as outgoing

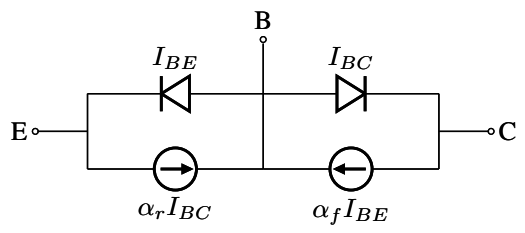

Fig. 12. Ebers Moll Model. (e.g. see Fig. 13(a) , so that the EMM is described by the system of equations:

$$
\left\{\begin{array}{l}
I_{E}=I_{B E}-\alpha_{r} I_{B C} \\
I_{C}=I_{B C}-\alpha_{f} I_{B E} \\
I_{E}+I_{B}+I_{C}=0
\end{array}\right.
$$

where

$$
I_{B E}=I_{E s}\left(e^{\frac{V_{B E}}{\eta V_{t}}}-1\right), I_{B C}=I_{C s}\left(e^{\frac{V_{B C}}{\eta V_{t}}}-1\right),
$$

being $I_{E s}$ and $I_{C s}$ the saturation currents of the two diodes described by the Shockley model (6). The BJT can be characterized by different numbers of PSTs, depending on the topology of the reference circuit. The presence of feedback can increase the number of ports up to 6 (16): 3 port voltages being potentials at terminals $\left(V_{C}, V_{B}\right.$ and $\left.V_{E}\right)$ and 3 port voltages being differences of potentials between terminals $\left(V_{C E}=V_{C}-V_{E}, V_{C B}=V_{C}-V_{B}\right.$ and $\left.V_{E B}=V_{E}-V_{B}\right)$. Adding feedback currents to the classical EMM, we obtain an Extended Ebers-Moll model (EEMM) of the BJT with 6 PSTs (named $E, B, C, C E, C B$ and $E B$ ):

$$
\left\{\begin{array}{l}
I_{E}=I_{B E}-\alpha_{r} I_{B C}+I_{C E}-I_{E B} \\
I_{C}=I_{B C}-\alpha_{f} I_{B E}-I_{C E}-I_{C B} \\
I_{E}+I_{B}+I_{C}=0,
\end{array}\right.
$$

where $I_{C E}, I_{E B}$ and $I_{C B}$ are the port currents passing through the loads of the three feedback ports. We can employ SMs to approximate the EMM and the EEMM. If the Collector-Base junction is reverse-biased the corresponding diode is removed. The same is done if the Emitter-Base junction is reversebiased. It can be verified that a parametrization leading to an explicit model based on the $W$ function can be found only for SMs of the EEMM with up to 3 ports. Such 3 ports can be either the 3 terminal-ground ports $E, C$ and $B$, as in Fig. 13 , or 2 of them and a feedback port having the 2 "active" terminals as prongs, as in Fig. 15. We notice that if one of the 3 potentials at terminals is zero, we end up with a degenerate case, since the remaining 2 terminal-ground ports and the 2 corresponding feedback ports have their respective pairs of prongs in common, as in Fig. 17. In Table I we show the parameterizations that can be used for finding explicit wave mappings of some SMs of the EEMM in relevant BJT amplifier configurations. In each row of Table II is specified which port variable is chosen as dependent variable $y$ and also the expression of the other parameters in eq. 3.

1) Common Collector BJT Amplifier: A 3-port NLE is used for implementing the BJT in the amplifier of Fig. 13 The first SM of Table I is employed together with a "specular" SM describing the BJT when the Emitter-Base junction is reversebiased. $V_{\text {bias }}$ is set to $0 \mathrm{~V}$ in order to test the amplifier in cutoff mode. The other parameter values of the simulation are $g_{\text {in }}=1.5 \mathrm{~V}, f_{0 i n}=200 \mathrm{~Hz}, R_{\text {in }}=1 \Omega, V_{g}=15$ $\mathrm{V}, R_{g}=2 \Omega, R_{\text {out }}=5000 \Omega, V_{t}=2510^{-3} \mathrm{~V}, \eta=1$, $I_{E s}=I_{C s}=4510^{-14} \mathrm{~A}, \alpha_{f}=0.9$ and $\alpha_{r}=0.8$. Fig. 14 shows the voltage across $R_{\text {out }}$, comparing the WD model output with a SPICE simulation. We notice that the contribute of the reversed biased Collector-Base junction is not completely negligible and this fact results in a smaller amplitude of the output voltage in SPICE. 
TABLE I

BJT SimPLIFIEd MODELS IN COMMON AMPLIFIER CONFIGURATIONS

\begin{tabular}{|c|c|c|}
\hline \multicolumn{2}{|c|}{ Simplified Model } & inear Dependences among Port Voltages or Port Currents and Gen \\
\hline $\begin{array}{l}\text { BJT with a reverse biased } \\
\text { Collector-Base junction } \\
\text { in a Common Collector } \\
\text { (or Emitter Follower) am- } \\
\text { plifier configuration im- } \\
\text { plemented as a 3-port } \\
\text { NLE. }\end{array}$ & $\overbrace{\mathrm{E} !}^{\mathrm{C}} \alpha_{f_{B E} I_{B E}} I^{\mathrm{C}}$ & $\begin{array}{l}y=I_{E}=I_{B E} \quad I_{B}=\left(\alpha_{f}-1\right) y \quad I_{C}=-\alpha_{f} y \\
\theta=\frac{R_{0 B}\left(\alpha_{f}-1\right)-R_{0 E}}{\eta V_{t}} \quad \beta=\frac{a_{B}}{\eta V_{t}}-\frac{a_{E}}{\eta V_{t}} \quad c=\frac{1}{I_{E s}} \quad d=1 \quad p=e \\
b_{E}=a_{E}+2 R_{0 E} I_{E} \quad b_{B}=a_{B}+2 R_{0 B} I_{B} \quad b_{C}=a_{C}+2 R_{0 C} I_{C}\end{array}$ \\
\hline $\begin{array}{l}\text { BJT with a reverse } \\
\text { biased Collector-Base } \\
\text { junction in a Common } \\
\text { Emitter amplifier with } \\
\text { feedback configuration } \\
\text { implemented as a 3-port } \\
\text { NLE. }\end{array}$ & $\underset{\mathrm{E}}{\bar{z}}{ }^{I_{B E}}$ & $\begin{array}{l}y=V_{B} \quad V_{C}=\frac{y\left(\frac{\alpha_{f}}{R_{0 B}}+\frac{1}{R_{0 C B}}\right)+a_{C}\left(\frac{1-\alpha_{f}}{R_{0 C}}\right)-\frac{a_{B} \alpha_{f}}{R_{0 B}}+\frac{a_{C B}}{R_{0 C B}}}{\left(1-\alpha_{f}\right) / R_{0 C}+1 / R_{0 C B}} \quad V_{C B}=V_{C}-y \\
\theta=\frac{1}{\eta V_{t}} \quad p=e \quad c=\frac{-1}{I_{E s}}\left(\frac{1}{R_{0 B}}+\frac{\alpha_{f} / R_{0 B}+1 / R_{0 C B}}{1-\alpha_{f}+R_{0 C} / R_{0 C B}}\right) \\
d=\frac{1}{I_{E s}}\left(\frac{a_{B}}{R_{0 B}}\left(\frac{\alpha_{f}}{1-\alpha_{f}+R_{0 C} / R_{0 C B}}+1\right)+\frac{a_{C}}{R_{0 C}}\left(\frac{\alpha_{f}-1}{1-\alpha_{f}+R_{0 C} / R_{0 C B}+1}\right)-\frac{a_{C B} / R_{0 C B}}{1-\alpha_{f}+\frac{R_{0 C}}{R_{0 C B}}}\right)+1 \\
b_{B}=2 V_{B}-a_{B} \quad b_{C}=2 V_{C}-a_{C} \quad b_{C B}=2 V_{C B}-a_{C B}\end{array}$ \\
\hline
\end{tabular}

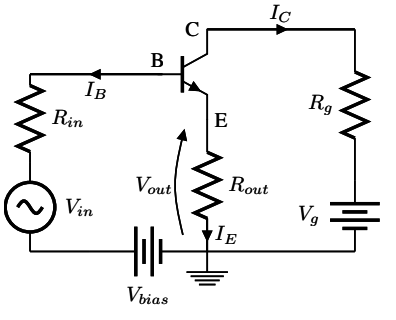

(a) Schematic

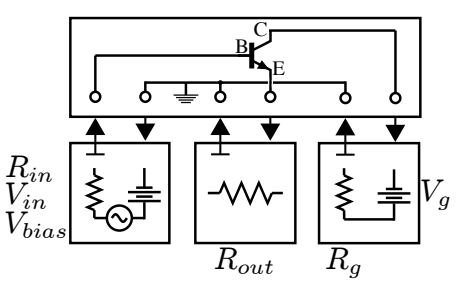

(b) WDS
Fig. 13. BJT Common Collector Amplifier.

2) Common Emitter BJT Amplifier with Base-Collector Feedback: A 3-port NLE employing both the second and the third SM of Table 1 is used for implementing the BJT in the amplifier of Fig. 15. The simulation parameters are: $g_{i n}=1.5$ $\mathrm{V}, f_{0 i n}=200 \mathrm{~Hz}, R_{\text {in }}=1000 \Omega, V_{\text {bias }}=2.3 \mathrm{~V}, V_{g}=15$ $\mathrm{V}, R_{g}=1 \Omega, R_{f B C}=400 \Omega$ and $R_{\text {out }}=8 \Omega$. The missing values are the same of V-D1. Fig. 16 shows the output current through $R_{\text {out }}$. Also in this case the contribute of the reversed
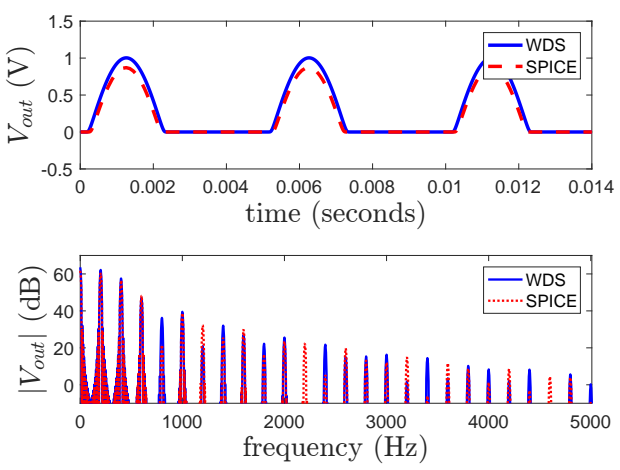

Fig. 14. BJT Common Collector Amplifier output voltage in the time domain and in the frequency domain (WDS vs. SPICE).

biased Collector-Base junction is not negligible and it results in a larger amplitude of the output current in SPICE.

3) Common Base BJT Amplifier: A 2-port NLE employing the fourth SM of Table Пis used for implementing the amplifier 


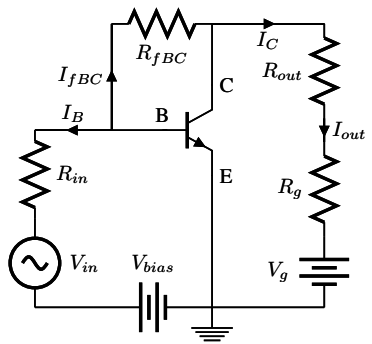

(a) Schematic

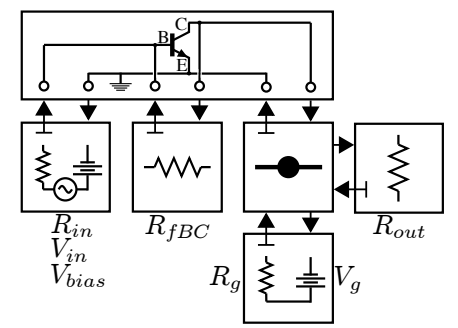

(b) WDS
Fig. 15. BJT Common Emitter Amplifier with base-collector feedback.
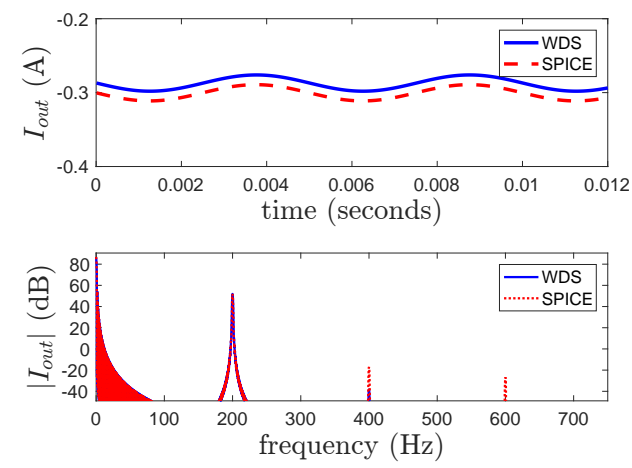

Fig. 16. BJT Common Emitter Amplifier output current in the time domain and in the frequency domain (WDS vs. SPICE).

of Fig. 17. The simulation parameters are: $g_{i n}=5 \mathrm{~V}$,

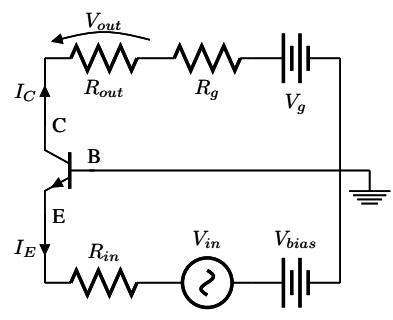

(a) Schematic

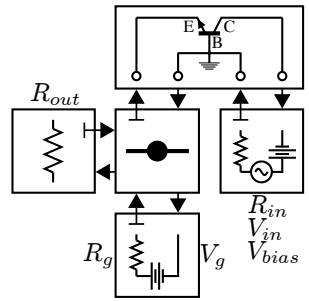

(b) WDS
Fig. 17. BJT Common Base Amplifier.

$f_{0 i n}=200 \mathrm{~Hz}, R_{\text {in }}=15 \Omega, V_{\text {bias }}=-12.5 \mathrm{~V}, V_{g}=90$ $\mathrm{V}, R_{g}=15 \Omega$ and $R_{\text {out }}=85 \Omega$. The missing values are the same of V-D1. Fig. 18 shows the voltage across $R_{\text {out }}$. In this case the mismatch w.r.t. to SPICE is smaller, since in the Common-Base configuration, the contribute of the reverse biased Emitter-Base junction has a low impact on $V_{\text {out }}$.

\section{CONCLUSIONS AND FUTURE WORK}

In this work we explored the use of the Lambert $W$ function in the WD domain for explicitly modeling exponential NLEs. We derived a new one-port wave mapping describing an arbitrary number of different parallel and antiparallel diodes. We also defined a new family of explicit WD models of multiport NLEs. We tested our models in a number of applications, including a transformerless ring modulator. We also introduced the novel 6-port EEMM model of the BJT, which allows us to describe the BJT in all the amplifier configurations that
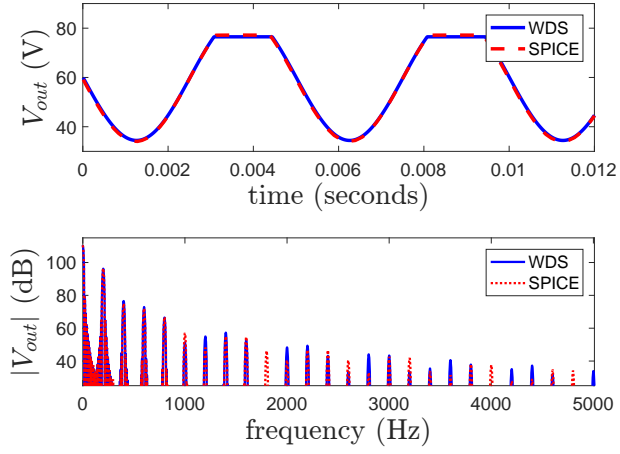

Fig. 18. BJT Common Base Amplifier output voltage in the time domain and in the frequency domain (WDS vs. SPICE).

include feedback. Comparing our results to SPICE outputs we found second order mismatches, as we are forced to trade some accuracy for the possibility of finding a closed-form solution. Although perfect waveform reconstruction is not always required in virtual analog modeling, we need to be careful about unwanted artifacts, as they could be perceivable. As far as future developments are concerned, we are planning to widen the family of WD multi-port NLEs in such a way to include exponential NLEs with memory.

\section{REFERENCES}

[1] A. Fettweis, "Wave digital filters: Theory and practice," Proc. of the IEEE, vol. 74, pp. 270-327, Feb. 1986.

[2] F. Pedersini, A. Sarti, and S. Tubaro, "Object-based sound synthesis for virtual environments using musical acoustics," IEEE Signal Process. Mag., vol. 17, pp. 37-51, Nov. 2000.

[3] G. De Sanctis and A. Sarti, "Virtual analog modeling in the wave-digital domain," IEEE Trans. Audio, Speech, Language Process., vol. 18, pp. 715-727, May 2010.

[4] A. Fettweis, "Pseudo-passivity, sensitivity, and stability of wave digital filters," IEEE Trans. Circuit Theory, vol. 19, pp. 668-673, Nov. 1972.

[5] A. Fettweis and K. Meerkötter, "Suppression of parasitic oscillations in wave digital filters," IEEE Trans. Circuits and Systems, vol. CAS-22, pp. 239-246, March 1975.

[6] R. C. D. de Paiva, "Circuit modeling studies related to guitars and audio processing," Ph.D. diss., Aalto University, Espoo, Finland, Sept. 2013.

[7] M. Holters and U. Zölzer, "Physical modeling of a wah-wah effect pedal as a case study for application of the nodal dk method to circuits with variable parts," in Proc. 14th Conf. Digital Audio Effects, Paris, France, Sept. 19-23 2011.

[8] K. Meerkötter and R. Scholz, "Digital simulation of nonlinear circuits by wave digital filter principles," in IEEE Int. Symp. Circuits Syst., vol. 1, June 1989, pp. 720-723.

[9] A. Sarti and G. De Poli, "Toward nonlinear wave digital filters," IEEE Trans. Signal Process., vol. 47, pp. 1654-1668, June 1999.

[10] S. Petrausch and R. Rabenstein, "Wave digital filters with multiple nonlinearities," in Proc. European Signal Process. Conf. (EUSIPCO), vol. 12, Vienna, Austria, Sept. 6-10 2004, pp. 77-80.

[11] R. C. D. Paiva, S. D'Angelo, J. Pakarinen, and V. Välimäki, "Emulation of operational amplifiers and diodes in audio distortion circuits," IEEE Trans. Circuits Syst. II, Exp. Briefs, vol. 59, pp. 688-692, Oct. 2012.

[12] V. Välimäki, S. Bilbao, J. O. Smith, J. S. Abel, J. Pakarinen, and D. Berners, Virtual Analog Effects, 2nd ed. John Wiley \& Sons, Ed. Udo Zölzer, 2011, ch. 12, pp. 473-522.

[13] A. Bernardini, K. J. Werner, A. Sarti, and J. O. Smith, "Multi-port nonlinearities in wave digital structures," in Proc. IEEE Int. Symp. Signals Circuits Syst. (ISSCS), Iasi, Romania, July 9-10 2015, pp. 1-4.

[14] M. Karjalainen and J. Pakarinen, "Wave digital simulation of a vacuumtube amplifier," in Proc. IEEE Int. Conf. on Acoust., Speech, Signal Process., vol. 5, Toulouse, France, May 14-19 2006, pp. 153-156.

[15] J. Pakarinen, M. Tikander, and M. Karjalainen, "Wave digital modeling of the output chain of a vacuum-tube amplifier," in Proc. Int. Conf. on Digital Audio Effects, Como, Italy, Sept. 1-4 2009. 
[16] J. Pakarinen and M. Karjalainen, "Enhanced wave digital triode model for real-time tube amplifier emulation," IEEE Trans. Audio, Speech, Language Process., vol. 18, pp. 738-746, May 2010.

[17] S. D'Angelo, J. Pakarinen, and V. Välimäki, "New family of wavedigital triode models," IEEE Trans. Audio, Speech, Language Process., vol. 21 , pp. 313-321, Feb. 2013.

[18] A. Bernardini, K. J. Werner, A. Sarti, and J. O. Smith, "Modeling a class of multi-port nonlinearities in wave digital structures," in Proc. European Signal Process. Conf. (EUSIPCO), Nice, France, Aug. 31 Sept. 4 2015, pp. 664-668.

[19] K. J. Werner, V. Nangia, J. O. Smith, and J. S. Abel, "A general and explicit formulation for wave digital filters with multiple/multiport nonlinearities and complicated topologies," in Proc. IEEE Workshop on Applicat. of Signal Process. to Audio and Acoust. (WASPAA), New Paltz, NY, Oct. 18-21 2015, pp. 1-5.

[20] R. C. D. de Paiva, J. Pakarinen, V. Välimäki, and M. Tikander, "Realtime audio transformer emulation for virtual tube amplifiers," EURASIP J. on Advances in Signal Process., pp. 688-692, Jan. 2011.

[21] A. Sarti and G. De Sanctis, "Systematic methods for the implementation of nonlinear wave-digital structures," IEEE Trans. Circuits Syst. I, Reg. Papers, vol. 56, pp. 460-472, Feb. 2009.

[22] D. Fränken, J. Ochs, and K. Ochs, "Generation of wave digital structures for connection networks containing multiport elements," IEEE Trans. Circuits and Systems I: Regular Papers, vol. 52, pp. 586-596, 2005.

[23] K. J. Werner, V. Nangia, J. O. Smith, and J. S. Abel, "Resolving wave digital filters with multiple/multiport nonlinearities," in Proc. 18th Conf Digital Audio Effects, Trondheim, Norway, Nov. 30 - Dec. 32015.

[24] K. J. Werner, J. O. Smith, and J. S. Abel, "Wave digital filters adaptors for arbitrary topologies and multiport linear elements," in Proc. 18th Conf. Digital Audio Effects, Trondheim, Norway, Nov. 30 - Dec. 32015.

[25] R. Corless, G. Gonnet, D. Hare, D. Jeffrey, and D. Knuth, "On the Lambert W function," Advances in Computational Math., vol. 5, pp. 329-359, Dec. 1996.

[26] T. C. Banwell and A. Jayakumar, "Exact analytical solution for current flow through diode with series resistance," Electron. Lett., vol. 36, pp. 291-292, Feb. 2000.

[27] T. C. Banwell, "Bipolar transistor circuit analysis using the Lambert W-function," IEEE Trans. Circuits Syst. I, Fundam. Theory Applicat., vol. 47, pp. 1621-1633, Nov. 2000.

[28] D. Veberic, "Having fun with Lambert W(x) function," GAP-2009-114. [Online]. Available: http://arxiv.org/abs/1003.1628.

[29] A. Ortiz-Conde, F. García-Sánchez, and J. Muci, "Exact analytical solutions of the forward non-ideal diode equation with series and shunt parasitic resistances," Solid-State Electronics, vol. 4, pp. 1861-1864, July 2000.

[30] A. Jain and A. Kapoor, "Exact analytical solutions of the parameters of real solar cells using Lambert W-function," Solar Energy Materials and Solar Cells, vol. 81, pp. 269-277, Feb. 2004.

[31] A. Ortiz-Conde and F. García-Sánchez, "Extraction of non-ideal junction model parameters from the explicit analytic solutions of its iv characteristics," Solid-State Electronics, vol. 49, pp. 465-472, March 2005.

[32] B. Romero, G. d. Pozo, and B. Arredondo, "Exact analytical solution of a two diode circuit model for organic solar cells showing S-shape using Lambert W-functions," Solar Energy, pp. 3026-3029, July 2012.

[33] H. Bayhan and A. Kavasoglu, "Exact analytical solution of the diode ideality factor of a pn junction device using Lambert W-function model," Turkish J. of Phys., pp. 7-10, June 2006.

[34] W. Jung and M. Guziewicz, "Schottky diode parameters extraction using Lambert W-function," Materials Sci. and Eng.: B, vol. 165, pp. 57-59, Nov. 2009.

[35] D. Lugo-Munoz, J. Muci, A. Ortiz-Conde, F. García-Sánchez, M. de Souza, and M. A. Pavanello, "An explicit multi-exponential model for semiconductor junctions with series and shunt resistances," IEEE $J$. of Photovoltaics I, vol. 2, pp. 261-268, July 2011.

[36] A. Ortiz-Conde, D. Lugo-Munoz, and F. García-Sánchez, "An explicit multiexponential model as an alternative to traditional solar cell models with series and shunt resistances," Microelectronics Reliability, vol. 51, pp. 2044-2048, July 2012.

[37] K. J. Werner, V. Nangia, A. Bernardini, J. O. Smith, and A. Sarti, "An improved and generalized diode clipper model for wave digital filters," in Proc. 139th Conv. Audio Eng. Soc., New York, NY, Oct. 29 - Nov. 12015.

[38] J. Parker, "A simple digital model of the diode-based ring-modulator," in Proc. Int. Conf. on Digital Audio Effects, Paris, France, Sept. 19-23 2011.

[39] J. J. Ebers and J. L. Moll, "Large-signal behavior of junction transistors," Proc. of the IRE, vol. 42, pp. 1761-1772, Dec. 1954.

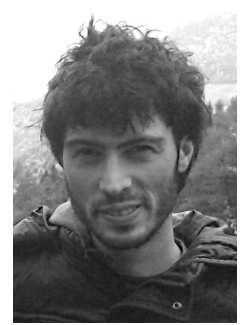

Alberto Bernardini received his B.S. from the University of Bologna, Italy, in 2012; and his M.S. degree (cum laude) from the Politecnico di Milano, Italy, in 2015, both in Computer Engineering. He is currently a Ph.D. candidate in Information Engineering at the Dipartimento di Elettronica, Informazione and Bioingegneria of the Politecnico di Milano, Italy. His main research interests are audio signal processing and modeling of nonlinear acoustic systems.

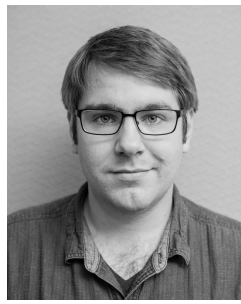

Kurt James Werner received his B.S. in General Engineering (w/ a secondary field in Acoustics) and a Bachelor of Music in Theory and Composition, both from the University of Illinois at UrbanaChampaign (UIUC). His technical research is focused on physical modeling and virtual analog audio processing, especially theoretical aspects of Wave Digital Filters. He is currently a Ph.D. candidate in Computer-Based Music Theory and Acoustics at Stanford University's Center for Computer Research in Music and Acoustics (CCRMA).

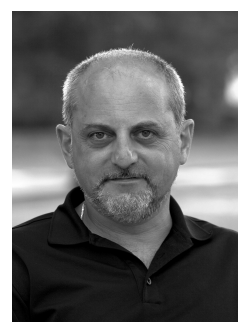

Augusto Sarti received his M.S. in electronic engineering from the University of Padua, Italy, in 1988. He then received his Ph.D. in Information Engineering in 1993, again from the University of Padua, Italy. His graduate studies were in a joint graduate program with the University of California, Berkeley. In 1993, he joined the faculty of the Politecnico di Milano, Italy. In 2013, he also joined the University of California, Davis, as an Adjunct Professor. His research interests are in the area of multimedia signal processing, with particular focus on sound analysis, synthesis and processing; space-time audio processing; geometrical acoustics; and music information extraction. He has also worked on problems of image analysis and 3D vision. He coauthored over 250 scientific publications on international journals and congresses as well as numerous patents in the multimedia signal processing area. He coordinates the activities of the Musical Acoustics Lab and of the Sound and Music Computing Lab of the Politecnico di Milano. He has been the promoter/coordinator and/or contributor to numerous (20+) European projects. He is an active member of the IEEE Technical Committee on Audio and Acoustics Signal Processing, and is in the Editorial Board of the IEEE.

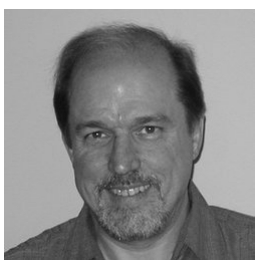

Julius Orion Smith III received the B.S.E.E. degree from Rice University, Houston, TX, in 1975 (control, circuits, and communication). He received the M.S. and Ph.D. degrees in E.E. from Stanford University, Stanford, CA, in 1978 and 1983, respectively. His $\mathrm{Ph} . \mathrm{D}$. research was devoted to improved methods for digital filter design and system identification applied to music and audio systems. From 1975 to 1977, he worked in the Signal Processing Department at ESL, Sunnyvale, CA, on systems for digital communications. From 1982 to 1986, he was with the Adaptive Systems Department at Systems Control Technology, Palo Alto, CA, where he worked in the areas of adaptive filtering and spectral estimation. From 1986 to 1991, he was employed at NeXT Computer, Inc., responsible for sound, music, and signal processing software for the NeXT computer workstation. After NeXT, he became an Associate Professor at the Center for Computer Research in Music and Acoustics (CCRMA) at Stanford, teaching courses and pursuing research related to signal processing techniques applied to music and audio systems. Continuing this work, he is presently Professor of Music and (by courtesy) Electrical Engineering at Stanford University. For more information, see http://ccrma.stanford.edu/jos/. 